\title{
Estruturação de um Banco de Dentes Humanos
}

\section{Structuralization of a Human Teeth Bank}

\author{
Alessandra Cristina da Silva Nassif* \\ Fabio Tieri** \\ Patricia Aparecida da Ana** \\ Sérgio Brossi Botta** \\ José Carlos Pettorossi Imparato***
}

\begin{abstract}
RESUMO: Um Banco de Dentes Humanos (BDH) é uma instituição sem fins lucrativos, vinculada a uma faculdade, universidade ou outra instituição. Seu propósito é suprir as necessidades acadêmicas, fornecendo dentes humanos para pesquisa ou para treinamento laboratorial pré-clínico dos alunos, dessa forma eliminando o comércio ilegal de dentes que ainda existe nas faculdades de Odontologia. Cabe também ao BDH zelar pela eliminação da infecção cruzada que existe no manuseio indiscriminado de dentes extraídos. Para o bom funcionamento de um BDH, é fundamental um controle severo de seus procedimentos internos, que incluem a separação e o estoque de dentes, assim como o cadastro e o arquivamento das fichas dos doadores ou beneficiários. Aqui, abordaremos as funções que um BDH pode desempenhar e a forma pela qual hoje o Banco de Dentes Humanos da Faculdade de Odontologia da Universidade de São Paulo (FOUSP) funciona e organiza-se.

DESCRITORES: Banco de dentes humanos; Organização e administração.
\end{abstract}

\begin{abstract}
A Human Teeth Bank (HTB) is a nonprofit institution, associated to a college, to a university or to some other institution. Its purpose is to fulfill academic needs, by supplying human teeth for research or for preclinical laboratory training of students, thus eliminating the illegal commerce of teeth that still takes place in dental schools. It is also up to an HTB to work for the elimination of cross-infection caused by indiscriminate handling of extracted teeth. In order to work properly, an HTB should have a strict control of its internal procedures, which include separation and stocking of teeth, as well as an appropriate maintaining of donors' and beneficiaries' records. In this article, we discuss the functions that an HTB can perform, and how the Human Teeth Bank, School of Dentistry, University of São Paulo, works and organizes itself today.
\end{abstract}

DESCRIPTORS: Human teeth bank; Organization and administration.

Um Banco de Dentes Humanos (BDH) é uma instituição sem fins lucrativos, que deve estar vinculada a uma faculdade, universidade ou outra instituição. Seu propósito é suprir as necessidades acadêmicas, fornecendo dentes humanos para pesquisa ou atividades didáticas.

Dentro desse contexto, a instituição BDH passa a assumir importante função ética, eliminando o comércio ilegal de dentes que ainda existe nas faculdades de Odontologia. De fato, o dente é um órgão do corpo humano e, como tal, está submetido à Lei de Transplantes Brasileira (lei 9434 de 04/02/1997), a qual prevê pena de 3 a 8 anos de reclusão e multa para quem remover, post-mortem, órgãos, tecidos e partes do corpo humano de pessoas não identificadas. O Código Penal também prevê pena de 1 a 3 anos de reclusão para aqueles que violarem sepultura (Artigo 210) e o Conselho
Nacional de Saúde exige os termos de consentimento livre e esclarecido dos sujeitos como forma de "respeito à dignidade humana" (Resolução 196 de 10/10/1996).

Cabe também ao BDH zelar pela eliminação da infecção cruzada que existe no manuseio indiscriminado de dentes extraídos. O BDH é organizado de forma a relacionar-se apropriadamente com a faculdade, universidade ou outra instituição a qual pertence, respeitando-se suas necessidades e regulamentos. Não existe, entretanto, um formato definitivo que seja seguido por todos os BDHs existentes.

Uma tentativa de padronização de protocolos de organização e funcionalidade ainda pode ser debatida e tornar-se uma realidade futura. Aqui, abordaremos as funções que um BDH pode desempenhar e a forma pela qual hoje, o Banco de Dentes

\footnotetext{
* Mestranda em Odontopediatria pela Faculdade de Odontologia da Universidade de São Paulo (FOUSP), Membro do Banco de Dentes Humanos (BHD) da FOUSP - Divisão decíduos.

** Cirurgiões-dentistas pela FOUSP. Membros do BDH-FOUSP - Divisão permanentes.

*** Professor Assistente da Disciplina de Odontopediatria da FOUSP, Coordenador do BDH-FOUSP.
} 
Nassif AC da S, Tieri F, Ana PA da, Botta SB, Imparato JCP. Estruturação de um Banco de Dentes Humanos. Pesqui Odontol Bras 2003;17(Supl 1):70-4.

Humanos da Faculdade de Odontologia da Universidade de São Paulo (FOUSP) funciona e organiza-se.

\section{IMPLEMENTAÇ̃̃O DE UM BANCO DE DENTES}

Para que um BDH possa exercer suas funções regularmente, é necessário que o mesmo esteja vinculado a uma instituição de ensino, o que requer preferência por faculdades de Odontologia. Para tal, as normas de implementação devem seguir as estabelecidas pela instituição de ensino para a instalação de laboratórios. Não é necessário estatuto.

A normatização do BDH segue as definições propostas pela diretoria ou reitoria, quando então é indicado um coordenador responsável que seja, preferencialmente, um docente qualificado. Este coordenador será o representante do BDH em reuniões ou conselhos, assim como deverá sugerir uma equipe para gerenciar o $\mathrm{BDH}$, responsabilizando-se pela mesma.

As diretrizes do BDH serão definidas através de regimento interno, baseadas nos objetivos de tal instituição. Cada membro terá uma função específica em um grupo onde deverão participar também alunos de graduação.

\section{ORGANIZAÇÃO}

Atualmente os Comitês de Ética em Pesquisa (CEP) não aprovam pesquisas que utilizam dentes humanos cuja origem não seja comprovada ou legalizada. E esse é o principal objetivo para que cada faculdade de Odontologia possua um BDH estruturado e regulamentado, eliminando, assim, as "coleções de dentes".

$\mathrm{O}$ que realmente diferencia um $\mathrm{BDH}$ de uma "coleção de dentes" é a organização mais criteriosa e sua ampla funcionalidade. Neste aspecto, deve-se considerar que o BDH deverá fornecer dentes para toda e qualquer pesquisa a ser realizada na faculdade onde estiver instalado, além de fornecer dentes para treinamento laboratorial pré-clínico dos acadêmicos. Para tal, torna-se imperioso o controle severo dos procedimentos internos do BDH, onde se incluem a separação e o estoque de dentes, assim como o cadastro e o arquivamento das fichas dos doadores e/ou beneficiários.

\subsection{Recursos físicos}

Para a realização das funções do BDH, é necessário um laboratório e uma sala de suporte. O laboratório deverá ser construído de acordo com as normas de vigilância sanitária correntes. Os equipamentos necessários relacionam-se com a seleção, limpeza e estoque dos dentes, a saber: refrigeradores para estocagem dos dentes, bancada para seleção e limpeza dos dentes, pias, armários, materiais de biossegurança pessoal, soluções desinfetantes, ultra-som, instrumental, vidraria de laboratório, dentre outros materiais.

Para a administração, é conveniente que se tenha uma sala anexa composta de microcomputador, arquivo, fax, telefone, mesas, armários e demais materiais para escritório necessários.

\subsection{Recursos humanos}

Dentre os membros do BDH, destacam-se o Coordenador Geral, cuja função é desempenhada por um professor cirurgião-dentista indicado pela instituição. Sugere-se que o BDH tenha uma subdivisão para dentes decíduos e outra para dentes permanentes. Desta forma, deverá haver um coordenador adjunto para cada subdivisão.

Os demais membros deverão exercer funções específicas, tais como: secretaria, assessoria científica, coordenação de projetos, biblioteca, arquivo, biossegurança, consultoria, laboratório, controle de entrada e saída de dentes, almoxarifado, dentre outras funções. Tais membros poderão ser estudantes de graduação, pós-graduação ou estagiários.

\section{FUNCIONALIDADE}

Para o bom funcionamento do BDH, deverão ser elucidadas as prioridades do mesmo. Dentre as funções do BDH pode-se enumerar:

- valorização do dente como órgão e divulgação;

- arrecadação dos dentes;

- preparação dos dentes;

- cessão e/ou empréstimo de dentes;

- administração dos dados e registros;

- realização de pesquisas e atividades didáticas.

\subsection{Valorização do dente como órgão e divulgação}

$\mathrm{O} \mathrm{BDH}$, através de atividades educativas, palestras, cartazes e folders, deve procurar conscientizar, tanto a comunidade leiga quanto a científica, da importância cultural, bioética, social, legal e moral da existência de um BDH como um banco de órgãos. Deve-se ressaltar que o dente, como qualquer outro órgão do corpo humano, somente poderá ser doado com o consentimento do paciente ou responsável, o que é expresso, para o $\mathrm{BDH}$, através de um Termo de Consentimento Livre e Esclarecido (Anexo 1). 
Nassif AC da S, Tieri F, Ana PA da, Botta SB, Imparato JCP. Estruturação de um Banco de Dentes Humanos. Pesqui Odontol Bras 2003;17(Supl 1):70-4.

A divulgação do BDH é de fundamental importância para o seu crescimento e para o desenvolvimento de suas funções. Com a divulgação consegue-se valorizar a importância do dente, aumentar o número de doações e, conseqüentemente, o número de atividades realizadas com dentes (como pesquisas e estudos laboratoriais pré-clínicos) e diminuir o comércio de dentes. O BDH da FOUSP realiza trabalhos de divulgação em congressos, jornais, sites e escolas de primeiro grau, assim como realiza ampla campanha de divulgação e conscientização dentro da própria Faculdade de Odontologia da USP.

\subsection{Arrecadação}

O BDH deverá responsabilizar-se pela obtenção de uma quantidade de dentes que seja necessária para a demanda das instituições as quais o Banco de Dentes auxilia. Para isso, é importante manter diferentes fontes de arrecadação, o que pode ser conseguido através de parcerias. As fontes de arrecadação podem ser as mais variadas: clínicas particulares, postos de saúde, clínicas da própria faculdade ou instituição de ensino, hospitais, graduandos, pesquisadores e a população em geral.

Prioridade deve ser dada quanto à legalidade da origem dos dentes. Nos casos onde um ou mais dentes serão extraídos, deve-se perguntar ao paciente se ele aceita doar os dentes e informá-lo o destino dos mesmos e com qual finalidade tais dentes serão utilizados. Caso ele concorde, deverá ser solicitada a sua assinatura no Termo de Consentimento Livre e Esclarecido (Anexo 1). Esse termo é individual, ou seja, é um termo por paciente e ele pode ser usado em clínicas particulares, postos de saúde, clínicas de instituições de ensino e hospitais.

Outra forma de arrecadação de dentes é aquela proveniente de cirurgiões-dentistas que possuem dentes de coleções particulares. Neste caso, utiliza-se o Termo de Doação de Dentes Humanos de Cirurgiões-Dentistas (Anexo 2), onde o profissional estará doando todos os dentes que estiverem estocados em seu consultório, responsabilizando-se por sua origem.

Existe ainda uma terceira fonte de arrecadação de dentes humanos, a qual é proveniente da população em geral que possui dentes em casa e queira doá-los. Para este fim, utiliza-se o Termo de Doação de Dentes Humanos (Anexo 3).

\subsection{Preparação}

A preparação dos dentes que chegam ao BDH inclui as etapas de manipulação, seleção, estoca- gem e desinfecção e/ou esterilização. O protocolo de manipulação, seleção e armazenamento de dentes para o BDH da FOUSP encontra-se no Anexo 4.

É importante enfatizar o aspecto de ainda não se ter encontrado um método de esterilização ou uma solução desinfetante que não interfira, de algum modo, nas propriedades fisico-químicas dos dentes, o que pode vir a comprometer os resultados dos testes in vitro realizados com os dentes que recebem algum tipo de tratamento. Entretanto, deve-se ressaltar a importância de se manter o dente esterilizado, visto que, como todo órgão do corpo humano, o elemento dental é fonte de patógenos severos para o homem.

Comprovou-se o potencial de contaminação do dente extraído, o que causou grande preocupação das entidades de saúde dos Estados Unidos, dentre as quais se destacam a OSHA (Occupational Safety and Health Administration), os CDCs (Centers for Disease Control and Prevention - Division of Oral Health) e a AADS (American Association of Dental Schools). Estas organizações passaram a regulamentar a utilização de dentes humanos extraídos, assim como a padronizar uma metodologia adequada para a doação dos mesmos para as escolas de Odontologia. O BDH da FOUSP está desenvolvendo um protocolo de utilização racional e segura de dentes humanos, baseado nos protocolos americano e nas normas de biossegurança da Faculdade de Odontologia da USP, assim como nas novas pesquisas que estão em andamento pela equipe do BDH-FOUSP.

\subsection{Cessão e/ou empréstimo}

Sempre que um dente for retirado do BDH, o aluno/pesquisador deverá preencher uma ficha cadastral para que se tenha controle do destino do dente e, no caso das pesquisas, um projeto de pesquisa deverá estar anexado juntamente com o parecer favorável do Comitê de Ética em Pesquisa (CEP) para a realização do trabalho.

Para a emissão do parecer do CEP, o BDH providencia uma Declaração de Doação de Dentes para aquele determinado estudo. A exigência da aprovação dos trabalhos pelo CEP impede "perda" ou "desperdício" de dentes doados para trabalhos que posteriormente não venham a ser aprovados pelo CEP.

Para os alunos de graduação, o BDH empresta os dentes pelo período determinado pela(s) disciplina(s) solicitante(s). Neste caso, os alunos, devidamente cadastrados, deverão devolver os dentes ao final do término do prazo concedido pelo $\mathrm{BDH}$, 
no estado em que se encontrarem, para que possam ser reutilizados caso haja oportunidade.

\subsection{Administração}

A entrada e saída dos dentes em um Banco de Dentes deverá ser computada em fichas específicas, obtendo-se, assim, o controle do número de dentes em estoque. Nestas fichas, anota-se o tipo e o número de dentes que entram ou saem, assim como a data da movimentação.

Para evitar que os "estoques" de determinados grupos de dentes esgotem-se, o BDH estabelece que para cada grupo de dentes deverá ser mantido um "estoque mínimo" a ser respeitado. A politica do "estoque mínimo" consiste na manutenção de no mínimo 100 dentes de cada grupo armazenados e que, ao ser atingido, servirá como "sinal de alerta". A partir de então, aquele grupo de dentes não poderá mais ser doado até que seu estoque seja reposto em, no mínimo, $50 \%$.

Os termos de doação são registrados e arquivados, assim como todas as fichas cadastrais dos alunos e pesquisadores. Nestas fichas, encontra-se o número e o tipo de dentes doados e/ou emprestados, assim como a data de devolução dos mesmos em caso de empréstimos. Outrossim, o $\mathrm{BDH}$ da FOUSP arquiva os projetos científicos auxiliados pelo $\mathrm{BDH}$.

\subsection{Desenvolvimento de pesquisas e atividades didáticas}

$\mathrm{O}$ BDH poderá realizar pesquisas voltadas ao seu próprio desenvolvimento ou outras das mais diversas áreas do conhecimento técnico-científico da Odontologia. Deve-se ressaltar que há poucas pesquisas relacionadas com BDHs, e que este deverá ser um passo importante para estabelecer-se a funcionalidade dos mesmos.

O BDH da FOUSP também realiza atividades didáticas com crianças, ensinando-as e orientando-as sobre como proceder com os dentes decíduos exfoliados. Tais atividades poderão ser expandidas para os dentes permanentes, através de atividades realizadas em instituições de ensino.

\section{COLABORAÇÃO COM A FORMAÇÃO DE OUTROS BANCOS DE DENTES PELO BRASIL}

O BDH da FOUSP realiza consultoria para a formação de novos Bancos de Dentes, através de encontros, palestras, matérias em jornais e revistas científicas, além de via telefone e e-mail. Trata-se de uma atividade pioneira que propicia a troca de experiências entre pesquisadores e promove a me-

\section{TERMO DE CONSENTIMENTO LIVRE E ESCLARECIDO}

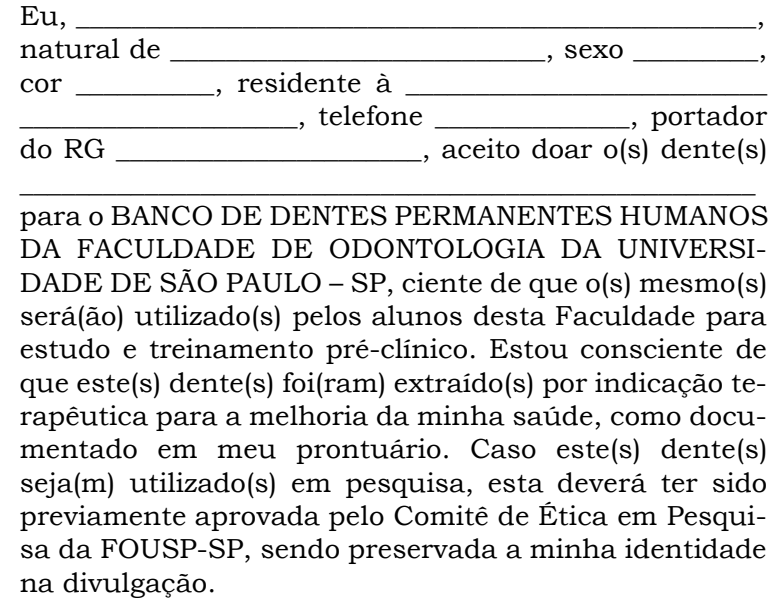
na divulgação.

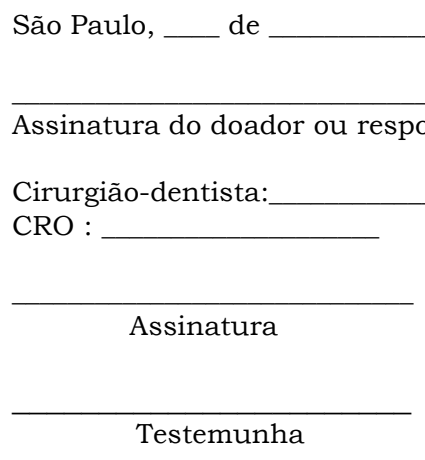
de 20

ANEXO 1

lhoria dos sistemas de Bancos de Dentes Humanos. O contato poderá ser feito através do e-mail: bdh@usp.br.

\section{ANEXOS}


Nassif AC da S, Tieri F, Ana PA da, Botta SB, Imparato JCP. Estruturação de um Banco de Dentes Humanos. Pesqui Odontol Bras 2003;17(Supl 1):70-4.

TERMO DE DOACÃO DE DENTES HUMANOS DE CIRURGIÕES-DENTISTAS

Eu,

cirurgião-dentista, inscrito no $\mathrm{CRO}$ , com consultório situado na bairro cidade , UF CEP telefone , dôo

dentes para o Banco de Dentes Permanentes Humanos - FOUSP, declarando que estes dentes foram extraidos por indicação terapêutica, cujos históricos fazem parte dos prontuários dos pacientes de quem se originam, arquivados sob minha responsabilidade. Estou ciente de que estes dentes serão utilizados pelos alunos e pesquisadores desta faculdade para treinamento laboratorial pré-clínico e realização de pesquisas.

São Paulo, de de 20

\section{Assinatura}

\section{ANEXO 2}

\section{TERMO DE DOAÇÃO DE DENTES HUMANOS}

Eu,

do $\mathrm{RG}$

de

telefone

portador estes dentes serão utilizados pelos alunos e pesquisadores desta faculdade para treinamento laboratorial pré-clínico e realização de pesquisas.

Origem dos dentes:

São Paulo, de de 20

\section{PROTOCOLO PARA ARMAZENAMENTO DOS DENTES DO BDPH-FOUSP}

Todos os dentes recém-chegados ao BDPH-FOUSP deverão seguir o protocolo assim especificado:

a) lavagem prévia com água corrente, detergente e esponja;

b) distribuição em recipientes específicos e identificados:

São separados em:

1. incisivos (superiores e inferiores)

2. caninos

3. pré-molares (superiores e inferiores)

4. molares (superiores e inferiores)

5. terceiros molares

6. raizes residuais

7. próteses (dentes com coroas totais, com raizes integras ou não)

8. anômalos

9. seccionados

c) armazenar todos os elementos em água destilada sob refrigeração, que deverá ser trocada semanalmente;

d) o número de dentes deve ser atualizado baseando-se no fluxo de entrada e saída;

e) identificar os recipientes com as etiquetas, com as seguintes anotações: data de chegada dos dentes (separados mensalmente), data da última troca da solução de armazenamento e tipo de dente;

f) raspagem dos dentes: remoção de restos orgânicos.

Ao total, serão 9 (nove) recipientes devidamente identificados.

Obs: A retirada ou a chegada de dentes, assim como a identificação dos mesmos, deverá ser anotada em fichas específicas para controle de entrada e saida dos dentes, devendo constar a assinatura do responsável pelo estoque de dentes.

Assinatura

ANEXO 4

ANEXO 3 